\title{
Did Germinal Centers Evolve Under Differential Effects of Diversity vs Affinity?
}

\author{
Jose Faro $^{1,2}$, Jaime Combadao ${ }^{1}$, and Isabel Gordo ${ }^{1}$ \\ ${ }^{1}$ Estudos Avançados de Oeiras, Instituto Gulbenkian de Ciência, Apartado 14, \\ 2781-901 Oeiras, Portugal \\ jfaro@igc.gulbenkian.pt, combadao@igc.gulbenkian.pt, \\ igordo@igc.gulbenkian.pt \\ ${ }^{2}$ Universidade de Vigo, Edificio de Ciencias Experimentáis, \\ Campus As Lagoas-Marcosende, 36310 Vigo, Spain \\ jfaro@uvigo.es
}

\begin{abstract}
The classical view on the process of mutation and affinity maturation that occurs in GCs assumes that their major role is to generate high affinity levels of serum Abs, as well as a dominant pool of high affinity memory B cells, through a very efficient selection process. Here we present a model that considers different types of structures where a mutation selection process occurs, with the aim at discussing the evolution of Germinal Center reactions. Based on the results of this model, we suggest that in addition to affinity maturation, the diversity generated during the GC reaction may have also been important in the evolution towards the presently observed highly organized structure of GC in higher vertebrates.
\end{abstract}

\section{Introduction}

Vertebrates have evolved a complex immune system (IS) that efficiently contributes to protect them from many infectious and toxic agents. To cope with such large variety of agents the IS generates a large diversity of lymphocyte receptors. This occurs through various mechanisms activated during lymphocyte development. The first one consists in the random recombination of relatively few gene segments into a full variable (V) region gene of immunoglobulins(Ig) heavy and light chains, allowing the formation of many different receptors [1]. In higher vertebrates (birds, mammals) the relevance of this mechanism for diversity generation in the primary B-cell repertoire varies with different species, being followed in some of them by other mechanisms like V-region gene conversion or somatic hypermutation (SHM) that act on rearranged V-region genes [2]. This initial repertoire is submitted to selection before B cells reach full maturity, thus getting purged of overt self-reactivity [1].

During an immune response to a protein antigen $(\mathrm{Ag})$ the SHM mechanism is triggered in some of the responding, mature B cells. Most mutations are deleterious (decrease the antibody (Ab) affinity for $\mathrm{Ag}$ ) or neutral, but a few may increase the affinity [3. This is followed by an increase of serum affinity starting 
after about the peak of the immune response until it reaches a quasi-plateau several weeks later [4. This process, termed affinity maturation, implies that a selection process for higher affinity Abs takes place during that time. In higher vertebrates the SMH and selection processes take place at Germinal Centers (GC) [2] These are short-lived structures, generated within primary follicles of secondary lymphoid tissue by migration of Ag-activated lymphocytes, and characterized by intense proliferation and apoptosis of Ag-specific B cells. In contrast, lower vertebrates do not generate GCs [2] so that SHM during immune responses to protein Ags takes place more or less diffusely in lymphoid tissue. Correspondingly in them the serum affinity during immune responses increases significantly less than in higher vertebrates. This indicates a less efficient selection process, currently attributed to their lack of GCs 2.

A higher rate affinity maturation process requires a more efficient (stronger) selection than a poorer affinity maturation process. On the other hand, the higher the efficiency the more specific the selected Abs will be, but the lower the remaining diversity related to the triggering Ag. However, thinking in evolutionary terms, keeping the diversity in the Ab repertoire seems at least as important as having the ability to selectively expand B cells producing Abs with higher specificity. For instance, while a 'selection structure' (i.e., GCs) has been selected for in higher vertebrates, many lower vertebrates have life spans similar to many higher vertebrates. Also, mutant mice that lack an enzyme essential for the SHM process get strong intestinal inflammation due to massive infiltration of normal anaerobic gut flora [5].

Because the more efficient the selection the less the diversity, and because of the importance of both affinity maturation and diversity, a trade-off between those two goals possibly emerged during the evolution of vertebrates in those species endowed with the physiologic possibility to generate GC-like structures. We hypothesize that such trade-off may have determined the size, life span, organization, etc. of GCs. In order to approach this issue, we have developed a simple stochastic/CA hybrid model that allows us to compare the degree of affinity maturation and diversity generated in different scenarios, intended to represent evolutionary stages of species with increasing GC size. In this model the process of affinity maturation within GCs is formally equivalent to a population genetics model of the evolution of clonal populations under mutation and selection. This allows us to put our findings in context with a number of analytical results from population genetics.

\section{The Model}

A model of the immune response incorporating SHM and selection, in which lymphoid tissue is represented by a $25 \times 25$ square grid with periodic boundary conditions, was implemented in language $C$. In it B cells are assumed to be distributed evenly in the small squares of the grid and are modeled as a large population with many subpopulations of equal size named demes. More specifically, each single square holds a deme of $N_{d}$ B cells (thus the whole system 
contains $N_{t}=N_{d} \times 625 \mathrm{~B}$ cells). Individual B cells are defined by strings representing V-regions with 300 nucleotides in size. The processes of SHM/selection take place only in particular demes named MS demes. Cells can migrate from one deme to any of the 8 neighbour demes with probability $m_{r}$ (see arrows in figure 1).

In each time step (generation) B cells within MS demes mutate in the $\mathrm{V}$ region of their Igs with rate $U$ per B cell per generation. The number of mutations occurring per cell is a Poisson random variable with mean $U$. Once a mutation occurs it can either decrease (with probability $p_{d}$ ) or increase (with probability $1-p_{d}$ ) the affinity of targeted Abs.

Outside of the MS demes, mutation does not occur and all cells have the same probability of survival. In the MS demes the probability of survival for each cell is directly proportional to its fitness $W_{i j}$, which depends on the affinity of its Igs for the Ag. $W_{i j}$ corresponds to the probability of survival of a $\mathrm{B}$ cell with $i$ mutations that decrease the affinity and $j$ mutations that increase affinity. To calculate the fitness of each B cell, we use the multiplicative fitness assumption for the interaction between mutations. With this assumption the fitness of $\mathrm{B}$ cells containing $i$ low affinity and $j$ high affinity mutations is calculated as: $W_{i j}=\left(1+s_{b}\right)^{j}\left(1-s_{d}\right)^{i}$, where $s_{b}$ is the effect of mutations that lead to an increase in affinity and $s_{d}$ is the effect of mutations that lead to a decrease in affinity.

To understand how different degrees of 'GC' aggregation/organization could affect the process of affinity maturation and the resulting diversity, five topologies were considered. These topologies are used to model different sizes of 'GC' represented by different areas where SHM and selection could take place. These were meant to model the evolution of GC size along a phylogenetic scale, going from vertebrates species where the SHM and affinity maturation did occur in less structured lymphoid tissue, to current higher vertebrates where these processes take place in finely organized GC structures. We have considered the following topologies (in figure 1 an example of the grid corresponding to topology A3 is shown): (i) topology A1 consists of 64 single, unconnected MS demes; (ii) topology A2 consists of 16 groups of $2 \times 2$ MS demes; (iii) topology A3 consists of 7 groups of $3 \times 3$ MS demes; (iv) topology A4 consists of 4 groups of $4 \times 4$ MS demes; and $(v)$ topology A5 consists of 1 group of $8 \times 8$ MS demes.

Each group of MS demes is placed at random in the grid. The simulations were performed using the following set of parameter values. Each deme is assumed to hold $N_{d}=100 \mathrm{~B}$ cells (this number is adjusted every generation, after the migration process has occurred). Within MS demes the mutation parameters are $U=0.3$ and $p_{d}=0.998$, and the selection parameters, $s_{d}$ and $s_{b}$, were varied. The migration rate was set to $m_{r}=0.00625$. This Monte-Carlo algorithm was run for different periods of time. In particular, analyses of the time for the mean affinity to approach the expected equilibrium were performed. To relate the time steps in the algorithm with the time scale of present day GCRs, we assume that B cells in the MS demes divide every 8 hours [3]. Thus 60 time steps in the algorithm correspond to about 21 days, which is the average life of GCs 


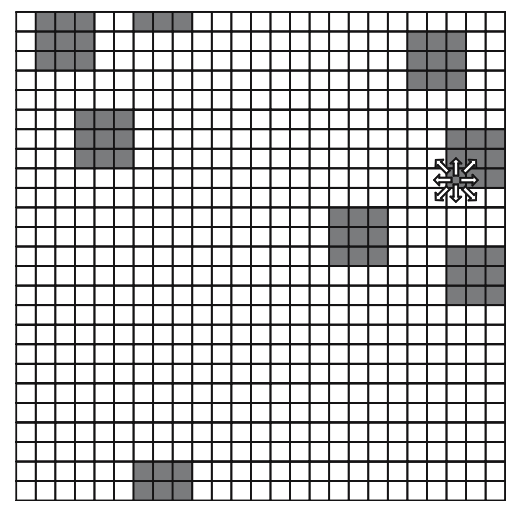

Fig. 1. An example of the $25 \times 25$ grid with a possible A3 topology. The full squares (MS demes) indicate the places where mutation and selection occur. Arrows indicate the eight possible directions for a migration event.

in primary immune responses. In order to obtain a variance due to stochastic events each simulation was repeated 20 times.

\section{Results}

\subsection{Some Results from Genetics Population Theory}

We first summarize some analytical results from population genetics that are relevant to understand the results shown for this model of $\mathrm{GC}$ evolution. Let us consider a large population of individuals (e.g., B cells) undergoing mutation at rate $U_{d}$ per individual per generation. Lets assume that every mutation has a negative effect, decreasing the fitness ( $\propto$ affinity) by an amount $s_{d}$. Then, after approximately $1 / s_{d}$ generations (each constituting a cycle of mutation and selection), the distribution of bad mutations in the population is Poisson with mean $U_{d} / s_{d}$. This means two things: first, if $s_{d}$ is small it takes a lot of time to achieve this distribution; second, when it is achieved it can have a very large mean and variance. In the simulations $s_{d}$ was around $10 \%$ the initial fitness so that the equilibrium distribution was reached in a period shorter than the time of a typical GC reaction of a primary immune response. Let $a(t)$ be the mean number of negative mutations at time $t$ after the start of the SHM process, then the distribution at time $t$ is Poisson with mean given by: $a(t)=\left(1-\left(1-s_{d}\right)^{t}\right) U_{d} / s_{d}$ 6]. Population genetics theory also shows that, if the population is not very large and/or $s_{d}$ is small, the equilibrium above is not stable and a continuous accumulation of deleterious mutations can occur [7]. This is likely to happen if the condition $N \times \operatorname{Exp}\left(-U_{d} / s_{d}\right)$ is satisfied, where $N$ is population size.

If positive (affinity increasing) mutations are allowed to occur at rate $U_{a}$ per cell per generation then for $U_{a} \ll U_{d}$ the distribution of negative mutations (decreasing affinity or deleterious) stays close to a Poisson [8]. 


\subsection{Average Affinity Increases with Aggregation Until a Plateau Is Reached}

We were interested in how 'affinity' (fitness) levels vary with the level of aggregation, that is, how 'affinity' levels vary with the size of the structure where the GCR occurs. Figure 2 shows the results for different values of the effect of mutations that increase and decrease affinity and for different times of the GCR. When considering short periods for the GCR, the average level of 'affinity' is low, even lower than the germ-line level of 'affinity', which by definition is 1 . But as we consider longer periods, we observe that the level of affinity increases as the size of the structures increase. In particular, given sufficient time, above a given size of the structures, the level of affinity reaches a plateau. This qualitative result is independent of the exact values of the selection parameters $s_{d}$ and $s_{b}$.

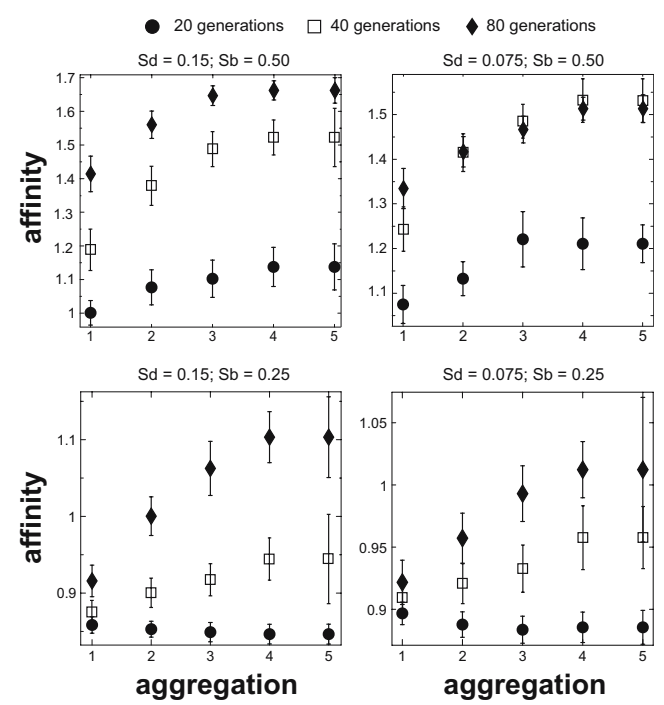

Fig. 2. Level of Ab affinity for increasing levels of aggregation at different times of the $\mathrm{GC}$ reaction

The reasons for this behaviour are as follows. When the size of the (GC) structure is small, the number of cells within each structure that are undergoing mutation and selection is small, so the contribution of the stochastic effects to the process is large. This means that, in order for a key mutation to overcome the effects of drift, the increase in affinity of that mutation has to be extremely high. Otherwise, most likely the mutant will be lost by chance. Thus, unless $s_{b}$ is very strong, for low values of the aggregation the level of affinity is low. When the size of the aggregate is large the stochastic effects are small, and so the probability that the key mutation spreads is higher. From population genetics theory of simple models of mutation and selection we know that the effects of selection are more important than the effects of drift when $s_{b}>1 / N_{e}$, where $N_{e}$ is the effective population size [9]. In our 
model, since both beneficial and deleterious mutations can occur, the value of $N_{e}$ depends on the mutation rate and on $s_{d}[10$.

The above result suggests that there is a critical GC size that leads to a maximal level of affinity. GCs of sizes above this value do not lead to further improvements in affinity. We can also see that organisms in which the process of SHM/selection is spread out in tiny structures may not achieve high levels of affinity maturation. This is compatible with what is observed in lower vertebrates.

\subsection{Changes in Average Diversity with Aggregation}

Next we have studied how the GC size influences the level of diversity for the whole set of reactions. The diversity of the surviving cells is measured by counting the number of pair-wise differences in the Ig $\mathrm{V}$ sequences between two random clones sampled from the GC population.

Figure 3 shows the results for different values of the mutation effects $s_{d}$ and $s_{b}$ and for different times of the GC reaction. Obviously, for short reaction times the diversity level is low, but as time increases this level approaches equilibrium. This depends on the values of the parameters governing mutation and selection, as discussed in the previous section.

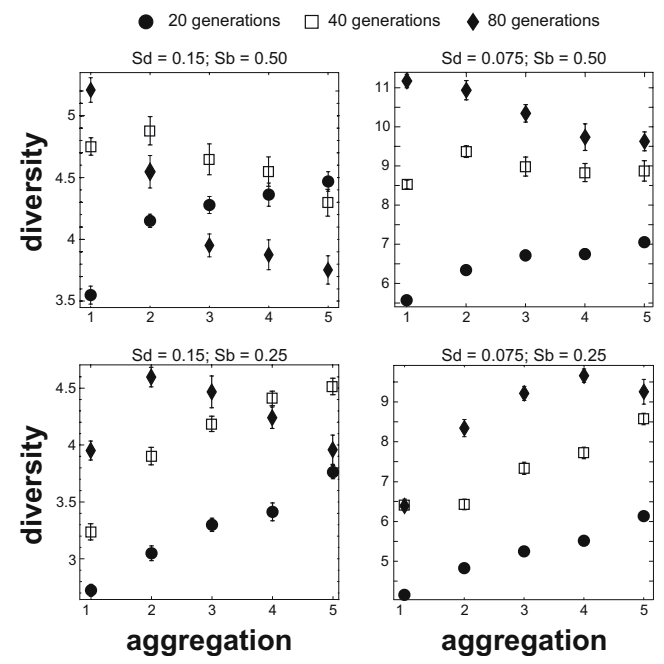

Fig. 3. Level of Ab diversity at different times of the GC reaction for increasing aggregation level

Initially the diversity generated is mainly due to deleterious mutations, but as time proceeds key mutations start to increase in frequency and they out-compete lower affinity clones. This may lead to an actual reduction in diversity. As larger aggregates lead to a higher probability of fixing key mutations the decrease in diversity is more pronounced for the larger aggregates. The wiping out of diversity in clonal populations is a well-established phenomenon in population 
genetics [1]. From this result we conclude that there is an intermediate value of the GC size for which the level of diversity generated is maximum.

Taken together, the above two results indicate that only GCs of some intermediate size lead to high levels of both affinity and diversity.

\section{Discussion}

The present preliminary results show that for lower values of aggregation, diversity and affinity maturation act together as a positive selection force for further aggregation increase. However, beyond a certain degree of aggregation there is a trade-off between diversity and affinity maturation. This leads to an optimal size of GCs, for which both high affinity Abs and a highly diverse pool of slightly different ones is produced. An important point that deserves mentioning is that the present results depend quantitatively on the particular definition of the fitness $W_{i j}$. However, we expect the qualitative behaviour will be much less affected by the fitness definition. On the other hand, the present multiplicative fitness definition of $W_{i j}$ is the most commonly used because of two major reasons: its simplicity and the fact that, as far as we know, to date there is no data relevant to establish a 'fitness landscape' linked to mutations affecting a particular phenotype, and in particular to those affecting the affinity of antibodies.

The classical view of GCs assumes that their major role is to generate high affinity levels of serum Abs, as well as a dominant pool of high affinity memory B cells, through a very efficient selection process [1]. However, in addition to affinity maturation, the diversity generated during the GCR may be also important. Two kind of experimental observations support this view. First, although all vertebrates display similar diversity generation by SHM during immune responses to protein Ags, lower vertebrates have significantly lower efficiency in selecting high affinity $\mathrm{Ab}$ mutants than higher vertebrates. However, lower and higher vertebrates have similar life spans. Second, mutant mice with impaired SHM get sick because of strong intestinal inflammation due to massive infiltration of normal anaerobic gut flora [5].

The preliminary results that we have presented here suggest an alternative view of the role of SHM in immune responses. According to it in present day higher vertebrates, the GC reaction not only facilitates the selection of high affinity mutant $B$ cells, but also allows for a rapid generation of (refined) diversity with the potential to recognize changes in the originally immunizing $\mathrm{Ag}$ (for instance, virus that mutate with high rate). In other words, the selection process may be only moderately efficient, and in some sense imperfect at leading to the creation of the best (highest affinity) possible memory B cell pool, but may have evolved just so to allow incorporation into the memory pool enough Ig diversity around the specificity of the initial triggered Igs. In this way different individuals can have a good coverage of the different mutational variants of a pathogen generated during its replication. That is, there would be an increased fitness for those individuals able to deal with pathogen variants, while conserving a large enough amount of Abs with increased affinity to the initial pathogen strain. We further speculate that the SHM mecha- 
nism could have co-evolved with mutational mechanisms in virus and bacteria focusing in each case in recognition molecules (e.g., Ig V regions in the first case and invasiveness molecules, like influenza hemaglutinin, in the second case), leading after a race similar high mutation rates and similar diversity generation compatible with the physiology of those molecules.

Many related important questions remain to be explored. What determines the SHM rate? Is it optimal? What determines the time of duration of the GCR? Under the view suggested above this time would be related not only to the mutation rate, but also to the diversity generated. For a given mutation rate, the diversity generated and the probability to spoil the physiologyof the Abs will increase with the duration of the GC reaction. Thus, the mutation rate and the duration of the mutational process will be the maximum compatible with preserving the role of the Abs, while the mutational mechanism of microorganisms must be limited also in their rates and the length of the period time in which it is active, being at rest in non-stressing environments.

\section{Acknowledgments}

The authors thank Jorge Carneiro and Joana Moreira for constructive comments on this work. This work was supported by Fundação para Ciência e Tecnologia, Portugal (grant to JF, POCTI/36413/1999, SFRH/BPD/8104/2002 to IG and $\mathrm{SFRH} / \mathrm{BD} / 5235 / 2001$ to JC). JF is supported by an Isidro Parga Pondal research contract by Xunta de Galicia, Spain.

\section{References}

1. Janeway, C., Travers, P., Walport, M., Shlomchik, M.: Immunobiology: the immune system in health and disease. 6th edn. Garland Science, New York (2004)

2. Flajnik, M.F.: Comparative analyses of immunoglobulin genes: surprises and portents. Nat Rev Immunol. 2 (2002) 688-698.

3. MacLennan, I. C.: Germinal centers. Annu. Rev. Immunol. 12 (1994) 117-139.

4. Kelsoe, G.: In situ studies of the germinal center reaction. Adv Immunol. 60 (1995) 267-288.

5. Fagarasan, S., Muramatsu, M., Suzuki, K., Nagaoka, H., Hiai, H. and Honjo, T.: Critical roles of activation-induced cytidine deaminase in the homeostasis of gut flora. Science 298 (2002) 1424-1427.

6. Gordo, I. and Dionisio, F.: Nonequilibrium model for estimating parameters of deleterious mutations. Phys Rev E Stat Nonlin Soft Matter Phys. 71 (2005) 031907.

7. Gordo, I. and Charlesworth, B.: On the speed of Muller's ratchet. Genetics. 156 (2000) 2137-2140.

8. Bachtrog, D. and Gordo, I.: Adaptive evolution of asexual populations under Muller's ratchet. Evolution Int J Org Evolution. 58 (2004) 1403-1413.

9. Kimura, M.: The neutral theory of molecular evolution. Cambridge University Press, Cambridge [Cambridgeshire] New York (1983)

10. Charlesworth, B., Morgan, M. T. and Charlesworth, D.: The effect of deleterious mutations on neutral molecular variation. Genetics. 134 (1993) 1289-1303.

11. Smith, J. M. and Haigh, J.: The hitch-hiking effect of a favourable gene. Genet Res 23 (1974) 23-35. 